\title{
Avifauna silvestre como recurso alimentar em áreas de semiárido no estado do Rio Grande do Norte, Brasil
}

\author{
Dandara Monalisa Mariz Bezerra ${ }^{1 *}$, Helder Farias Pereira de Araujo $^{2} \&$ Rômulo Romeu Nóbrega Alves ${ }^{3}$
}

${ }^{1}$ Departamento de Sistemática e Ecologia, Universidade Federal da Paraíba, Campus I, 58051-900, João Pessoa, Paraíba, Brasil.

${ }^{2}$ Departamento de Biologia, Universidade Federal da Paraíba, Campus II, 58397-000, Areia, Paraíba, Brasil.

${ }^{3}$ Departamento de Ciências Biológicas e da Saúde, Universidade Estadual da Paraíba, 58109-753, Campina Grande, Paraíba, Brasil.

\begin{abstract}
Resumo - Esta pesquisa foi desenvolvida junto aos moradores locais dos municípios de Caicó e São João do Sabugi, situados no Semiárido do estado do Rio Grande do Norte, Nordeste do Brasil. Teve por objetivo inventariar as aves utilizadas como recurso alimentar e caracterizar suas respectivas técnicas de captura. A pesquisa de campo foi realizada entre os meses de janeiro e fevereiro de 2010. Foram entrevistados 60 moradores locais que utilizavam ou interagiam com aves silvestres. A obtenção das informações ocorreu por meio de entrevistas semiestruturadas aplicadas individualmente. Foram registradas 13 espécies de aves silvestres distribuídas em seis ordens e seis famílias, sendo que a família Columbidae foi a mais representativa em número de espécies. A espécie Zenaida auriculata foi a que apresentou maior número de citações. As aves citadas são capturadas através de cinco técnicas: arapuca, espera, sangra, fôjo e rede, sendo a espera a técnica mais citada. $\mathrm{O}$ uso de aves silvestres na alimentação aliado à escassez de estudos da situação populacional de espécies cinegéticas e associado a outros problemas ambientais podem afetar direta ou indiretamente suas populações. Diante desse contexto, faz-se necessário a realização de mais estudos sobre essas temáticas, pois somente assim, poderse-á implantar de forma mais adequada programas de manejo e conservação interligados ao contexto sociocultural da região. Palavras-chave adicionais: aves cinegéticas, caatinga, caça de subsistência, conservação, etno-ornitologia.
\end{abstract}

\begin{abstract}
Wild birds as source of food in the semi-arid region of Rio Grande do Norte State, Brazil) - The present work was undertaken with the cooperation of locals in the municipalities of Caicó and São João do Sabugi in the semi-arid region of Rio Grande do Norte State, northeastern Brazil. We listed the wild birds used as sources of food in the region and described the techniques used to capture them. Field work was carried out between January and February of 2010, during which a total of 60 locals, that used or interacted with wild birds in some way, were interviewed. Information was obtained through semi-structured interviews from each individual. A total of 13 wild bird species, distributed among six orders and six families, were identified; the Columbidae family was the best represented in terms of the numbers of species. Zenaida auriculata was cited most. The birds were captured using five techniques: "arapuca" (a non-lethal fall-trap), "espera" (waiting in a blind), "sangra" (a non-lethal fall-trap), "fôjo" (dead-fall), and "rede" (netting); 'Waiting in a blind' was the technique most cited. The use of wild birds as sources of food, together with the general lack of population studies of hunting species is associated with other environmental problems that can be expected to directly or indirectly affect their populations. In this context, more studies should be undertaken focusing on these issues, because only then will it be possible to implement adequate wildlife management and conservation programs that can be linked to the socio-cultural context of the region.
\end{abstract}

Additional key words: caatinga, conservation, cynegetic birds, ethno-ornithology, subsistence hunting.

O conjunto complexo de interações que as culturas humanas mantêm com os animais pode ser abordado por meio de diferentes recortes científicos, a depender da linha teórica considerada (Begossi 1993) e do grupo taxonômico pesquisado (Alves \& Souto 2010). A interação humanos/ aves, é abordada pela perspectiva da Etno-ornitologia, termo que indica como um grupo étnico vê, percebe, classifica, nomeia e em geral se relaciona com as aves (Maxwell 1969). Segundo Farias \& Alves (2007), a Etnoornitologia busca compreender as relações cognitivas, comportamentais e simbólicas entre a espécie humana e as aves.

Autora para correspondência: dand.biologa08@gmail.com

Editor responsável: Marcelo Alves Ramos

Recebido: 16 maio 2011; aceito: 13 out. 2011.
A importância da avifauna silvestre para várias comunidades espalhadas pelo Brasil tem estimulado a realização de pesquisas etno-ornitológicas, que vêm investigando as formas de interação das aves com populações urbano-rurais. Muitas espécies de aves silvestres são utilizadas, no Brasil e em várias partes do mundo, para diversas finalidades, como por exemplo, uso em rituais místicos (Léo Neto et al. 2009; Léo Neto \& Alves 2010), no comércio ilegal (Ferreira \& Glock 2004; Pereira \& Brito 2005; Jepson \& Ladle 2005; Souza \& Soares Filho 2005; Rocha et al. 2006; Gama \& Sassi 2008), como animais de estimação (Balderas et al. 2001; Jepson \& Ladle 2005, 2009; Alves et al. 2009a, 2010a; Fernandes-Ferreira et al. 2010), na medicina popular (Costa-Neto \& Oliveira 2000; Alves et al. 2008, 2011; Mahawar \& Jaroli 2008; Ferreira et 
al. 2009) e na alimentação (Balderas et al. 2001; Casas et al. 2009; Hanazaki et al. 2009; Parry et al. 2009).

A importância do uso da avifauna por populações em áreas do Semiárido nordestino vem sendo evidenciada em trabalhos recentes (Alves et al. 2008; Alves et al. 2009a,b, 2010a; Ferreira et al. 2009; Barbosa et al. 2010; FernandesFerreira et al. 2010, 2012; Bezerra et al. 2011). As pessoas que vivem nessa área interagem com a biodiversidade local de diversas maneiras, principalmente praticando a caça de subsistência, que representa uma atividade passada de pai para filho e que faz parte da cultura da população local (Alves et al. 2010b). Segundo Olmos et al. (2005), a caça, tanto para obtenção de proteína como para suprir o comércio de aves de gaiola, praticamente extinguiu espécies como a zabelê (Crypturellus noctivagus zabele Spix, 1825), a graúna (Gnorimopsar chopi Vieillot, 1819), o canário (Sicalis flaveola Linnaeus, 1766), além dos psitacídeos maiores, e mantém reduzidas as populações regionais de aves normalmente abundantes em áreas pouco caçadas, como ocorre com Patagioenas picazuro (Temminck, 1813).

Um dos maiores desafios para conservação da vida silvestre na Caatinga consiste em integrar a conservação dos recursos naturais e as necessidades humanas (Alves et al. 2010b). A falta de políticas e de incentivo ao desenvolvimento sustentável tem contribuído para a concentração da pobreza e desigualdade social, alimentando o ciclo de degradação ambiental (Major et al. 2004). Alves et al. (2010a) apontam que, no Semiárido brasileiro, o uso de aves silvestres, apesar de ser uma prática ilegal, é bastante difundida e, em conjunto com outras ameaças, tem contribuído para o declínio da avifauna local. Assim, a persistência das atividades de caça certamente está interligada ao contexto cultural e socioeconômico. Nesta perspectiva, esta pesquisa teve como propósito inventariar as espécies da avifauna silvestre usadas como recurso alimentar, buscando também caracterizar as técnicas de captura destas espécies utilizadas por moradores de áreas localizadas no Semiárido do estado do Rio Grande do Norte, Nordeste do Brasil.

\section{Material e Métodos}

Área de estudo. A área de estudo desta pesquisa englobou os municípios de Caicó ( $06^{\circ} 27^{\prime} 28^{\prime \prime}$ 'S, 3705'52”W) e São João do Sabugi ( $06^{\circ} 43^{\prime} 04^{\prime}$ 'S, $37^{\circ} 12^{\prime} 03^{\prime}$ 'W), estado do Rio Grande do Norte (Figura 1). Os municípios pertencem à Mesorregião Central Potiguar e à Microrregião Seridó Ocidental e estão situados na Caatinga. Pela classificação de Köppen, o clima é Bswh, ou seja, seco, muito quente, com temperatura média anual de $27,5^{\circ} \mathrm{C}$ e estação chuvosa no verão (Bezerra Júnior \& Silva 2007). A população alvo do estudo incluiu moradores locais que utilizavam ou interagiam com aves silvestres de alguma forma.

Procedimentos de coleta e análise dos dados. A

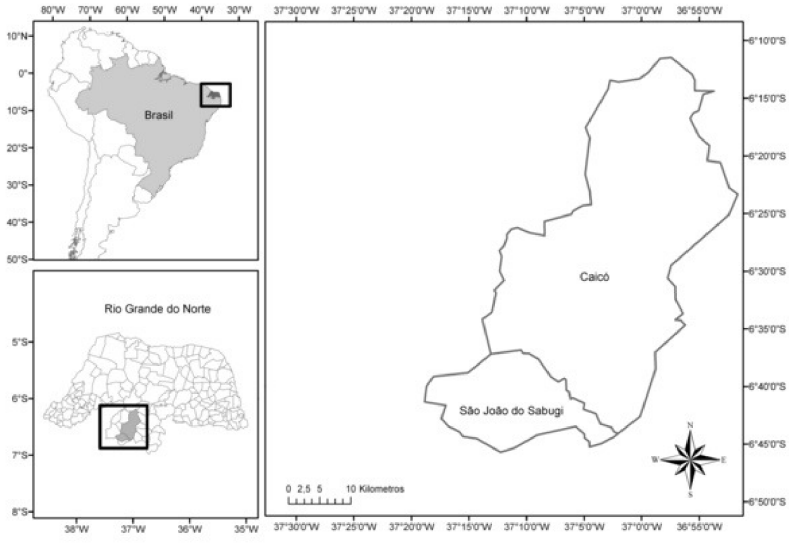

Figura 1. Mapa da área de estudo evidenciando os municípios de Caicó e São João do Sabugi.

pesquisa foi realizada entre os meses de janeiro e fevereiro de 2010. As informações foram obtidas através de entrevistas aplicadas a 60 moradores, distribuídos em dois municípios: Caicó $(\mathrm{n}=31)$ e São João do Sabugi $(\mathrm{n}=29)$. A escolha dos entrevistados foi através da técnica bola-deneve (Biernacki \&Waldorf 1981), que consiste em localizar os demais entrevistados alvos da pesquisa a partir da indicação dos primeiros.

As entrevistas foram realizadas individualmente e gravadas com aparelho MP3, sendo transcritas com máxima fidelidade e organizadas em um banco de dados padronizado. As entrevistas semiestruturadas abordavam aspectos sobre espécies de aves utilizadas como recurso alimentar e as formas de captura.

Realizaram-se testes de verificação de consistência e de validade das respostas (Marques 1991), recorrendo-se a entrevistas repetidas em situações sincrônicas e diacrônicas. As primeiras ocorrem quando uma mesma pergunta é feita a indivíduos diferentes em tempos bastante próximos e as segundas, quando uma pergunta é repetida a um mesmo indivíduo em tempos bem distintos.

Todas as aves citadas foram identificadas em nível específico, através de visualização direta durante as entrevistas e registros fotográficos, sendo utilizada bibliografia especializada (Perlo 2009; Ridgely \& Tudor 2009). A taxonomia utilizada neste estudo segue a classificação sugerida pelo Comitê Brasileiro de Registros Ornitológicos (CBRO 2011).

É importante salientar que foi explicado aos potenciais entrevistados, em linguagem acessível, os propósitos e objetivos gerais do estudo que tem aprovação do Comitê de Ética em Pesquisa do Hospital Universitário Lauro Wanderley na Universidade Federal da Paraíba (protocolo CEP/HULW no. 410/09).

Os dados qualitativos obtidos a partir das entrevistas estão sendo analisados por meio de uma abordagem emicista/eticista (Costa-Neto \& Marques 2000), na qual os conhecimentos foram comparados com aqueles 
correspondentes e/ou correlacionados na literatura científica. Estes dados, quando possível, também foram quantificados.

\section{Resultados E Discussão}

Foram entrevistadas 60 pessoas, seis do sexo feminino e 54 do sexo masculino com idades variando de 10 a 75 anos. Do total de entrevistados, $20 \%$ citaram espécies de aves silvestres utilizadas como recurso alimentar, enquanto os demais citaram aves usadas para outras finalidades, tais como animais de criação, zooterápicos e crenças. A pequena porcentagem de entrevistados que citaram espécies direcionadas à alimentação, provavelmente, deve-se às características da amostra, pois incluiu uma parcela significativa de pessoas $(n=49)$ residentes em áreas urbanas, que costumam ter o hábito de capturar aves para serem usadas como animais de estimação, enquanto os que residem em áreas rurais caçam as aves principalmente para fins alimentícios.

Aves silvestres utilizadas na alimentação. Foram registradas 13 espécies de aves silvestres distribuídas em seis ordens e seis famílias (Tabela 1). Os entrevistados costumam capturar para uso alimentar espécies das famílias Anatidae, Ardeidae, Cariamidae, Columbidae, Rallidae e Tinamidae. Para Santos \& Costa-Neto (2007), espécies dessas famílias geralmente têm em comum a grande massa corporal, o que implica na escolha destas quando o motivo da caça é a alimentação. Entretanto, nossos resultados apontaram que as quatro espécies do gênero Columbina, apesar de possuírem menor porte corporal, são utilizadas como fonte de proteína na área pesquisada, revelando que o tamanho da ave não é o único fator que influencia em sua escolha, mas provavelmente o sabor da carne e a disponibilidade da espécie.

A família Columbidae foi a mais representativa em número de espécies, compreendendo sete, das 13 espécies citadas. Constatação similar foi encontrada por Alves et. al. (2009a) em estudo no Semiárido paraibano, sugerindo que esta família tem uma importância significativa como recurso trófico na região do Semiárido nordestino. Alves et al. (2009b) referem-se à avifauna como um recurso trófico importante para as comunidades do Semiárido nordestino. O abate de aves em práticas cinegéticas, não está associado apenas à subsistência, mas também pode ser revestido de um caráter esportivo (Alves et al. 2009b), muito embora o produto desse tipo de caçada seja consumido pelos caçadores ou doados para consumo entre amigos e familiares.

A arribaçã (Zenaida auriculata) foi a ave que apresentou maior número de citações (Figura 2) corroborando com o estudo de Barbosa et al. (2010), que apontaram esta ave como umas das mais citadas para consumo alimentar no município de Queimadas, Semiárido do estado da Paraíba, sendo superada apenas para outro columbídeo, a rolinha-cabocla (Columbina minuta). De acordo com a lista de espécies ameaçadas de extinção da IUCN (2010), a Z. auriculata encontra-se na categoria de baixa preocupação. No entanto, percebe-se a necessidade de mais estudos para confirmar a situação populacional desta ave, visto que sua carne é bastante apreciada no Nordeste brasileiro, sendo raros os estudos sobre a densidade da avifauna em ambientes de Caatinga. Além disso, trata-se de uma espécie migratória que realiza

Tabela 1. Lista das espécies utilizadas como recurso alimentar pelos moradores dos municípios de Caicó e São João do Sabugi, estado do Rio Grande do Norte.

Táxon $\quad$ Nome local

ANSERIFORMES - Anatidae

Dendrocygna viduata (Linnaeus, 1766)

Amazonetta brasiliensis (Gmelin, 1789)

CARIAMIFORMES - Cariamidae

Cariama cristata (Linnaeus, 1766)

COLUMBIFORMES- Columbidae

Columbina minuta (Linnaeus, 1766)

Columbina talpacoti (Temminck, 1811)

Columbina squammata (Lesson, 1831)

Columbina picui (Temminck, 1813)

Patagioenas picazuro (Temminck, 1813)

Leptotila verreauxi (Bonaparte, 1855)

Zenaida auriculata (Des Murs, 1847)

GRUIFORMES - Rallidae

Gallinula galeata (Lichtenteins, 1818)

PELECANIFORMES - Ardeidae

Ardea alba (Linnaeus, 1758)

TINAMIFORMES - Tinamidae

Nothura boraquira (Spix, 1825)

Marreca

Paturi

Sariema, Seriema

Rolinha-cabocla

Rolinha caldo-de-feijão

Rolinha-cascavelinha

Rolinha-branca

Asa-branca

Juriti

Arribaçã

Galinha-da-água

Garça

Codorniz

Sitientibus série Ciências Biológicas 11(2): 177-183. 2011. 


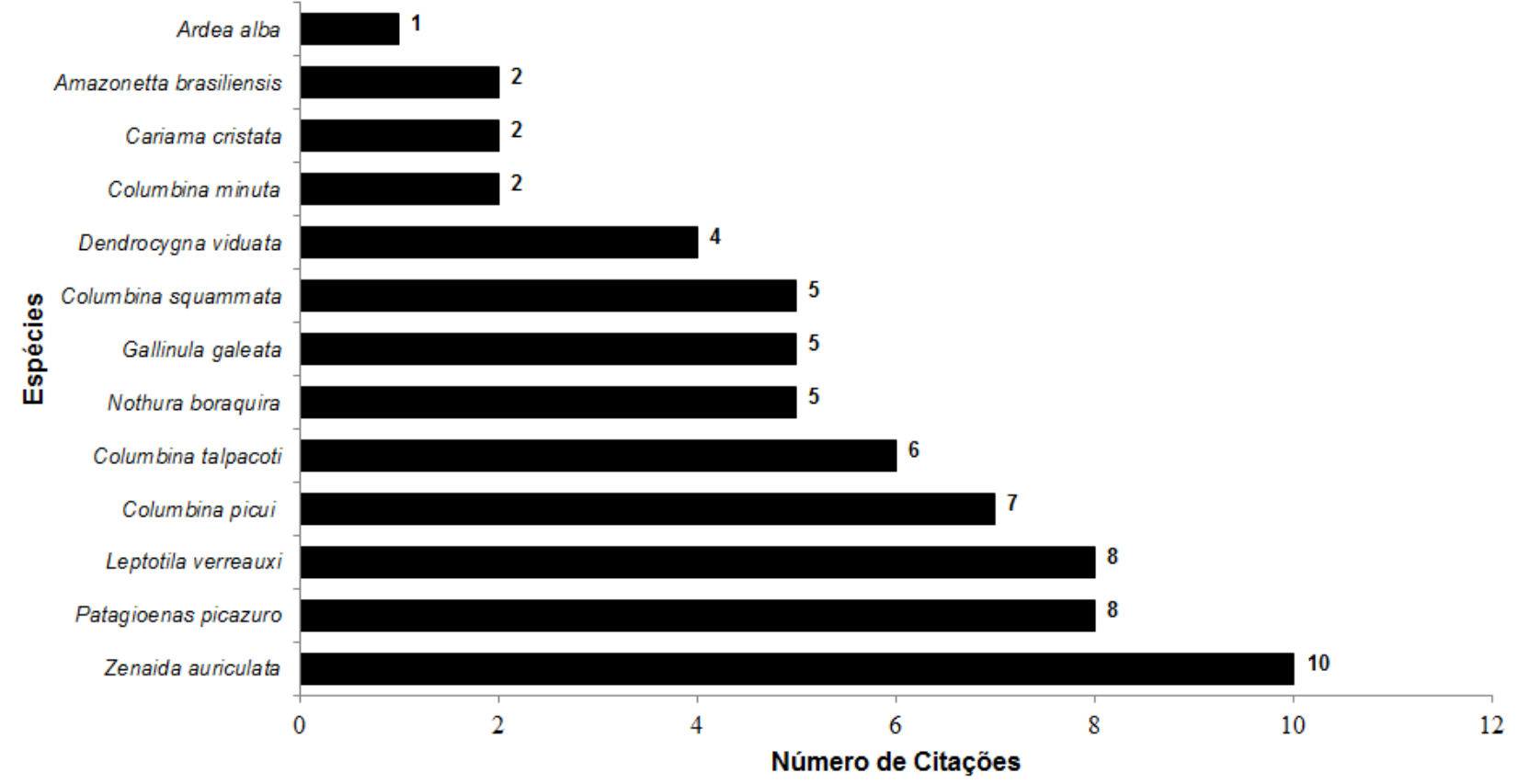

Figura 2. Espécies de aves silvestres utilizadas como recurso alimentar com seus respectivos número de citações pelos entrevistados de Caicó e São João do Sabugi no estado do Rio Grande do Norte.

deslocamentos no domínio da Caatinga de acordo com a disponibilidade de sementes e com o ritmo das chuvas (Olmos et al. 2005), e por isso é de extrema importância avaliar se a atividade cinegética não afeta seu período reprodutivo.

Técnicas de captura. Os depoimentos dos entrevistados evidenciam que as atividades cinegéticas iniciam-se ainda na infância. Nessa fase, o principal instrumento utilizado no abate das aves é a baladeira (estilingue). Quando adultos, os entrevistados utilizam diversas técnicas de captura, as quais são escolhidas conforme a espécie, hábitat, período do ano e uso a que se destinam. Foram citadas pelos entrevistados cinco técnicas de captura: arapuca, espera, sangra, fôjo e rede, sendo a espera a mais utilizada na captura de espécies destinadas a alimentação (Figura 3). Com exceção da rede, as demais técnicas também foram registradas em localidades do Semiárido paraibano (Alves et al. 2009a).

A variedade de técnicas registradas para captura de aves reflete a diversidade de espécies cinegéticas utilizadas pelas populações locais. Como apontado por Alves et al. (2009a), muitas espécies da Caatinga possuem hábitos caracterizados pela sazonalidade local. Assim, a utilização de várias técnicas é popularmente importante para que o caçador tenha sucesso na captura dos espécimes, sendo necessário que ele detenha informações acerca das características ecológicas das espécies, a exemplo de aspectos relacionados à alimentação, ao hábitat preferencial, ao período reprodutivo, entre outros.

Constatou-se a existência de técnicas especializadas para determinadas aves, enquanto outras podem ser utilizadas para uma ampla diversidade de espécies. A espera

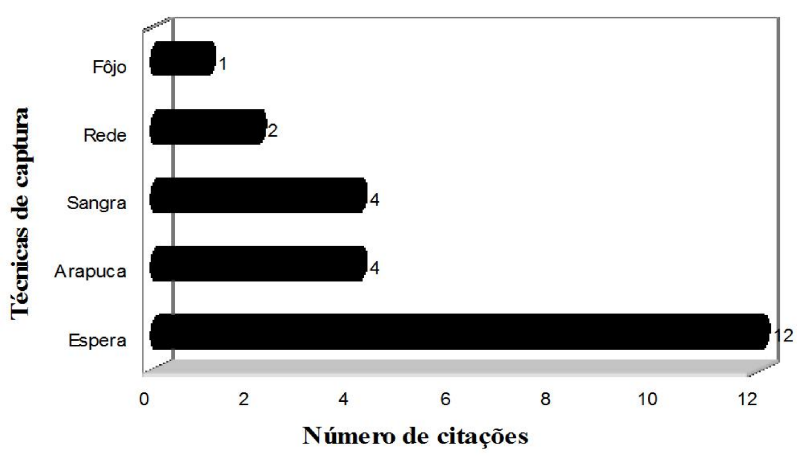

Figura 3. Técnicas de captura e número de citações pelos entrevistados de Caicó e São João do Sabugi, estado do Rio Grande do Norte.

é uma técnica mais generalista, pois permite a caça de uma maior diversidade de espécies, enquanto outras são restritas a somente algumas espécies, como por exemplo, a sangra e o fôjo, que são direcionadas especialmente à captura da arribaçã (Zenaida auriculata) e da codorniz (Nothura boraquira), respectivamente.

A técnica espera implica na construção de uma estrutura feita com galhos de plantas locais. Uma vez construída a espera, o caçador permanece em seu interior de tocaia, ficando totalmente camuflado entre os galhos das plantas e preparado para abater as aves que se aproximarem, usando para isto uma espingarda (arma de fogo) ou baladeira. Esta técnica consiste em uma estratégia do tipo "senta-e-espera". Geralmente os lugares escolhidos pelo caçador para montar a "espera" localizam-se nas adjacências de ambientes onde a ave costuma beber e/ou comer. O caçador também procura ambientes onde aves 
costumam empoleirar-se para dormir. A espera é utilizada para caçar as seguintes espécies: rolinha-cabloca (Columbina minuta), rolinha-caldo-de-feijão (Columbina talpacoti), rolinha-cascavelinha (Columbina squammata), rolinha-branca (Columbina picui), asa-branca (Patagioenas picazuro), juriti (Leptotila verreauxi), arribaçã (Zenaida auriculata), codorniz (Nothura boraquira), sariema (Cariama cristata). A espera é utilizada em conjunto com armas de fogo, e por isso são consideradas mais impactantes para a fauna local por serem mais eficientes (Alves et al. 2009a). As armas de fogo constituem um instrumento importante na caça, servindo tanto para o abate da presa como também para a proteção do próprio caçador (Alves et al. 2010b; Barbosa et al. 2010).

A arapuca consiste em uma armadilha com formato piramidal feita a partir da madeira do marmeleiro (Croton blanchetianus Baill.). A arapuca armada é suportada por uma forquilha e dois pedaços de madeiras em posição inclinada. Em seu interior colocam-se grãos de arroz, milho ou feijão como isca. Quando a ave adentra na arapuca com intuito de alimentar-se, a armadilha recai sobre o chão prendendo-a em seu interior. Está técnica pode ser utilizada para capturar as espécies: galinha-da-água (Gallinula. galeata), arribaçã (Zenaida auriculata), paturi (Amazonetta brasiliensis) e marreca (Dendrocygna viduata).

A sangra é uma armadilha também feita com varas de marmeleiro (C. blanchetianus), sendo semelhante à arapuca. No entanto, em uma das laterais existe uma abertura onde é colocado um "pente". Este consiste em uma estrutura com pontas em uma de suas extremidades, sendo construído a partir de um capim conhecido localmente por barba-de-bode (Poaceae). A parte do pente com as pontas fica voltada para o interior da sangra, de forma que permite a entrada da ave, mas impede sua saída. Para uso efetivo desta armadilha, inicialmente não se deve colocar o pente na sangra, sendo necessário colocar iscas (grãos de milho ou feijão) por três a cinco dias, dessa forma as aves ficam livres para entrar e sair, acostumando-se a estrutura da armadilha. Este processo é denominado pelos caçadores de "avezar". Depois desse período, o caçador coloca o pente na sangra e captura as aves que entrarem na armadilha.

O fôjo é armado cavando-se um buraco no solo e sobre este encaixa uma grade feita de madeira. Esta grade possui uma tábua central que cede quando ave caminha sobre ela, fazendo esta cair no interior do buraco, logo depois a tábua fecha impedindo a fuga da ave. Colocam-se sementes de milho próximo ao fôjo e em cima da tábua para atrair a ave. No Semiárido paraibano, Alves et al. (2009a) cita esta técnica sendo utilizada na captura de lambus (Crypturellus sp.) e de mamíferos de pequeno e médio porte.

A rede, que pode ser usada na captura da Dendrocygna viduata, consiste em uma rede de pesca de náilon e deve ser colocada na margem de um açude com arroz em palha debaixo dela como isca. Dessa forma quando a marreca for alimentar-se, os pés e a cabeça ficam presas na rede, facilitando a captura por parte do caçador.

Implicações conservacionistas. A caça de aves como recurso alimentar constitui uma fonte de proteína alternativa, gratuita e saborosa, sendo considerada uma iguaria pelos entrevistados. Adicionalmente, o divertimento na procura e captura dos espécimes também é um fator estimulador nas práticas cinegéticas desta região, evidenciando que aspectos socioeconômicos e culturais estão associados a tais práticas. Para Alves et al. (2010b), a persistência de atividades de caça no Brasil, apesar da ilegalidade, está intimamente associada a questões culturais e com o fato de que os animais cinegéticos têm grande importância nutricional para famílias de baixas renda. A pobreza da população e a destruição de habitats são considerados os principais desafios para a conservação da vida silvestre na Caatinga (Leal et al. 2005; Marini \& Garcia 2005; Olmos 2005), havendo a necessidade de priorizar investimentos para conciliar a conservação da biodiversidade e as necessidades humanas (Leal et al. 2005; Alves et al. 2010b).

De acordo com Olmos (2005), a caça de subsistência é considerada um problema sério para várias espécies da avifauna, a exemplo da jacucaca (Penelope jacucaca Spix, 1825), que consta na lista brasileira de espécies ameaçadas de extinção (Silveira \& Straube 2008). Um fator bastante relevante relacionado à atividade cinegética, diz respeito ao tipo de técnica utilizada, pois existem aquelas que são mais impactantes. Na presente pesquisa, foi constatada a preferência pela técnica da espera em detrimento a técnicas mais artesanais. De acordo com Alves et al. (2010b), a espera é mais impactante para a fauna cinegética do Semiárido por ser bastante eficiente. Esta eficiência está relacionada à quantidade de espécimes e à diversidade de aves que podem ser capturadas, bem como ao menor tempo gasto na captura.

As espécies registradas nesta pesquisa não constam na lista brasileira de aves ameaçadas de extinção (Silveira \& Straube 2008), e aparecem com baixo nível de preocupação na lista de espécies ameaçadas da IUCN (União Internacional para a Conservação da Natureza). Essas listas consideram a situação populacional das espécies em nível nacional e mundial. Entretanto, segundo Marini \& Garcia (2005), no caso do Brasil, poucos estados compilaram suas listas estaduais de espécies ameaçadas, as quais têm enorme valor na divulgação da situação da fauna, influenciando e direcionando as medidas de conservação. Assim, torna-se importante a elaboração de listas em escala mais regional, como forma de verificar a disponibilidade das populações de aves silvestres, sendo uma informação importante para as espécies que sofrem pressão cinegética.

Segundo Leal et al. (2005), não apenas a caça de animais silvestres, mais também atividades como a agricultura de corte e queima, o corte de madeira para lenha e a contínua remoção da vegetação para a criação de bovinos e caprinos, quando praticadas de forma não sustentável, têm levado ao empobrecimento em larga escala da biodiversidade da Caatinga. Portanto, no âmbito local, 
programas de educação ambiental, conservação e manejo sustentável da avifauna silvestre utilizada como recurso alimentar devem considerar o contexto sociocultural das pessoas envolvidas nesta atividade como também as demais pressões antrópicas no ambiente.

\section{Considerações Finais}

O uso da avifauna silvestre como recurso alimentar por moradores de áreas localizadas no Semiárido do estado do Rio grande do Norte representa uma prática enraizada na cultura das populações locais. Os resultados sugerem que a massa corporal, o paladar da carne e a disponibilidade local de aves silvestres sejam fatores que influenciam na escolha das espécies da avifauna nas áreas pesquisadas. Essas aves são capturadas com diferentes técnicas, sendo a espera preferida pela sua eficiência.
O uso de aves silvestres na alimentação, aliado à escassez de estudos sobre a situação populacional de espécies cinegéticas e a outros problemas ambientais, podem afetar direta ou indiretamente suas populações. Diante desse quadro, faz-se necessário a realização de mais estudos sobre essas temáticas, pois somente assim poderse-á implantar de forma mais adequada programas de manejo e conservação interligados ao contexto sociocultural da região.

\section{Agradecimentos}

À Coordenação de Aperfeiçoamento de Pessoal de Nível Superior (CAPES), pelo auxílio financeiro. A todos os informantes dos municípios de Caicó e São João do Sabugi, por toda a hospitalidade e colaboração para a realização desta pesquisa.

\section{REFERÊNCIAS}

Alves, R.R.N. \& Souto, W. 2010. Panorama atual, avanços e perspectivas futuras para etnozoologia no Brasil. In: R.R.N. Alves, W.M.S. Souto \& J.S. Mourão (orgs), A Etnozoologia no Brasil: importância, status atual e perspectivas. NUPEEA, Recife, p. 19-40.

Alves, R.M.S.; Lima, H.N.; Tavares, M.C.; Souto, W.M.S. \& Barboza, R.R.D.E. \& Vasconcellos, A. 2008. Animal-based remedies as complementary medicines in Santa Cruz do Capibaribe, Brazil. BMC Complementary and Alternative Medicine 8: 44.

Alves, R.R.N.; Mendonça, L.E.T.; Confessor, M.V.A.; Vieira, W.L.S. \& Lopez, L.C.S. 2009a. Hunting strategies used in the semi-arid region of northeastern Brazil. Journal of Ethnobiology and Ethnomedicine 5: 12.

Alves, R.R.N.; Neto, N.A.L.; Brooks, S.E. \& Albuquerque, U.P. 2009b. Commercialization of animal-derived remedies as complementary medicine in the Semi-arid Region of Northeastern Brazil. Journal of Ethnopharmacology, 124: 600-608.

Alves, R.R.N.; Nougueira, E.E.G.; Araujo, H.F.P. \& Brooks, S.E. 2010a. Bird-keeping in the Caatinga, NE Brasil. Human Ecology 38: 147-156.

Alves, R.R.N; Mendonça, L.E.T; Confessor, M.V.A.; Vieira, W.L.S; Vieira, K.S. \& Alves, F.N. 2010b. Caça no Semiárido paraibano: uma abordagem etnozoológica. In: R.R.N. Alves, W.M.S. Souto \& J.S. Mourão (orgs), A Etnozoologia no Brasil: importância, status atual e perspectivas. NUPEEA, Recife, p. 347-378.

Alves, R.R.N.; Barbosa, J.A.S.; Santos, S.L.D.; Souto, W.M.S. \& Barboza, R.R.D. 2011. Animal-based remedies as complementary medicines in the semi-arid region of northeastern Brazil. Evidence-based Complementary and Alternative Medicine: ID 179876.

Balderas, A.J.C.; Salas, J.A.G.; Velasco, A.G. \& Rojas, J.I.G. 2001. Aprovechamiento de las aves cinegéticas, de ornato y canoras de Nuevo León, México. Ciencia Universidad Autónoma de Nuevo León 4: 462-469.
Barbosa, J.A.A.; Nóbrega, V.A. \& Alves, R.R.N. 2010. Aspectos da caça e comércio ilegal da avifauna silvestre por populações tradicionais do Semiárido paraibano. Revista de Biologia e Ciências da Terra 10: 39-49

Begossi, A. 1993. Ecologia Humana: um enfoque das relações homem-ambiente. Interciencia 18: 121-132.

Bezerra Júnior, J.G.O. \& Silva, N.M. 2007. Caracterização geoambiental da Microrregião do Seridó Oriental do Rio Grande do Norte. Holos 2: 78-91.

Bezerra, D.M.M.S.Q; Araujo, H.F.P. \& Alves, R.R.N. 2011. The use of wild birds by rural communities in the semi-arid region of Rio Grande do Norte State, Brazil. Bioremediation, Biodiversity and Bioavailability 5: 117-120.

Biernacki, P.E. \&Waldorf, D. 1981. Snowball sampling problems and techniques of chain referral sampling. Sociological Methods and Research 10: 141-163.

Casas, F; Mougeot, F.; Viñuela, J. \& Bretagnolle, V. 2009. Effects of hunting on the behaviour and spatial distribution of farmland birds: importance of hunting-free refuges in agricultural areas. Animal Conservation 12: 346-354.

CBRO (Comitê Brasileiro de Registros Ornitológicos) 2011. Lista de Aves do Brasil. 9 ed. Disponível em: < http://www.cbro.org. br >; acesso em: 10 de jan. de 2011.

Costa-Neto, E.M. \& Marques, J.G.W. 2000. Conhecimento ictiológico tradicional e a distribuição temporal e espacial dos recursos pesqueiros pelos pescadores de Conde, estado da Bahia, Brasil. Etnoecológica 4: 56-67.

Costa-Neto, E.M. \& Oliveira, M.V.M. 2000. Cockroach is good for asthma: zootherapeutic practices in Northeastern Brazil. Human Ecology Review 7: 41-51.

Farias, G.B. \& Alves A.G.C. 2007. Aspectos históricos e conceituais da etnoornitologia. Biotemas 20: 91-100.

Fernandes-Ferreira, H.; Mendonça, S.V.; Albano, C.; Ferreira, F.S. \& Alves, R.R.N. 2010. Comércio e criação de aves silvestres (Psittaciformes, Piciformes e Passerifomes) no estado do Ceará. In: R.R.N Alves, W.M.S. Souto \& J.S. Mourão (orgs), A Etnozoologia no Brasil: importância, status 
atual e perspectivas. NUPEEA, Recife, p. 381-402.

Fernandes-Ferreira, H.; Mendonça, S.V.; Albano, C.; Ferreira, F. S. \& Alves, R.R.N. 2012. Hunting use and conservation of birds in Northeast Brasil. Biodiversity and Conservation 21: 221-244.

Ferreira, C.M. \& Glock, L. 2004. Diagnóstico preliminar sobre a avifauna traficada no Rio Grande do Sul, Brasil. Biociências 12: $21-30$.

Ferreira, F.S.; Brito, S.V.; Ribeiro, S.C.; Almeida, W.O. \& Alves, R.R.N. 2009. Zootherapeutics utilized by residents of the community Poço Dantas, Crato - CE, Brazil. Journal of Ethnobiology and Ethnomedicine 5: 1-10.

Gama, T.P. \& Sassi, R. 2008. Aspectos do comércio ilegal de pássaros silvestres na cidade de João Pessoa, Paraíba, Brasil. Gaia Scientia 2: 1-20.

Hanazaki, N.; Alves, R.R.N. \& Begossi, A. 2009. Hunting and use of terrestrial fauna used by Caiçaras from the Atlantic Forest coast (Brazil). Journal of Ethnobiology and Ethnomedicine 5: 36.

IUCN (International Union for Conservation of Nature) 2010. Lista Vermelha de Espécies Ameaçadas. Disponível em: www.iucnredlist.org; acesso 4 maio 2011.

Jepson, P \& Ladle, R.J. 2005. Bird-keeping in Indonesia: conservation impacts and the potential for substitution-based conservation responses. Oryx 39: 442-448.

Jepson, P \& Ladle, R.J. 2009. Governing bird-keeping in Java and Bali: evidence from a household survey. Oryx 43: 364 374.

Leal, I.R.; Silva, J.M.C.; Tabarelli M. \& Larcher, T.E.Jr. 2005. Changing the course of biodiversity conservation in the Caatinga of Northeastern Brazil. Conservation Biology 19: 701-706.

Léo Neto, N.A. \& Alves, R.R.N. 2010. A natureza sagrada do candomblé: análise da construção mística a cerca da natureza em terreiros de candomblé no Nordeste do Brasil. Interciências 35: 568-574.

Léo Neto, N.A.; Brooks, S.E. \& Alves, R.R.N. 2009. From Eshu to Obatala: animals used in sacrificial rituals at Candomblé "terreiros" in Brazil. Journal of Ethnobiology and Ethnomedicine 5: 23.

Mahawar, M.M \& Jaroli, D.P. 2008. Traditional zootherapeutic studies in India: a review. Journal of Ethnobiology and
Ethnomedicine 4: 17.

Major, I.; Sales Jr, L.G. \& Castro, R. 2004. Aves da Caatinga. Demócrito Rocha, Associação Caatinga, Fortaleza.

Marini, M.A. \& Garcia, F.I. 2005. Conservação de aves no Brasil. Megadiversidade 1: 95-102.

Marques, J.G.W. 1991. Aspectos Ecológicos na Etnoictiologia dos Pescadores do Complexo Estuarino-Lagunar MundaúManguaba. Tese de Doutorado. Universidade de Campinas.

Maxwell, A. R. 1969. Kedayan ethno-ornithology - a preliminary report. Brunei Museum Journal 1: 197-217.

Olmos, F. 2005. Aves ameaçadas, prioridades e políticas de conservação no Brasil. Natureza \& Conservação 3: 21-42.

Olmos, F.; Silva, W.A.G. \& Albano, C.G. 2005. Aves em oito áreas de caatinga no sul do Ceará e oeste de Pernambuco, Nordeste do Brasil: composição, riqueza e similaridade. Papéis Avulsos de Zoologia 45: 179-199.

Parry, L.; Barlow, J. \& Peres, C.A. 2009. Hunting for sustainability in Tropical Secondary Forest. Conservation Biology 23: 1270-1280.

Pereira, G.A. \& Brito, M.T. 2005. Diversidade de aves silvestres brasileiras comercializadas nas feiras livres da Região Metropolitana do Recife, Pernambuco. Atualidades Ornitológicas 126: 14.

Perlo, B.V. 2009. A Field Guide to the Birds of Brazil. Oxford University Press, London.

Ridgely R.S. \& Tudor, G. 2009. Field Guide to the Songbirds of South America: the passerines. University of Texas Press, Austin.

Rocha, M.S.P.; Cavalcanti, P.C.M.; Sousa, R.L. \& Alves, R.N.A. 2006. Aspectos da comercialização ilegal de aves nas feiras livres de Campina Grande, Paraíba, Brasil. Revista de Biologia e Ciências da Terra 2: 204-221

Santos, I.B. \& Costa-Neto, E.M. 2007. Estudo etnoornitológico em uma região do Semiárido do estado da Bahia, Brasil. Sitientibus série Ciências Biológicas 7: 273-288.

Silveira, L.F. \& Straube, F.C. 2008. Aves ameaçadas de extinção no Brasil. In: A.B Machado, G.M. Drummond \& A.P. Paglia. (orgs), Livro Vermelho da Fauna Brasileira Ameaçada de Extinção. Ministério do Meio Ambiente, Brasília, p. 379678.

Souza, G.M. \& Soares Filho, A.O. 2005. O comércio ilegal de aves silvestres na região do Paraguaçu e sudoeste da Bahia. Enciclopédia Biosfera 1:1-10. 